"This submitted manuscript has been authored by a contractor of the U.S. Govermment under Contract No. DE-ACO5-

960R22464. Accordingly, the U.S.

Government retains a nonexclusive, royalty-free license to publish or reproduce the published form of this contribution, or allow others to do so, for U.S. Government purposes."

\title{
The PVM 3.4 Tracing Facility and XPVM 1.1 *
}

\author{
James Arthur Kohl (kohl@msr.epm.ornl.gov) \\ G. A. Geist (geist@msr.epm.ornl.gov) \\ Computer Science \& Mathematics Division \\ Oak Ridge National Laboratory \\ Oak Ridge, TN 37831-6367
}

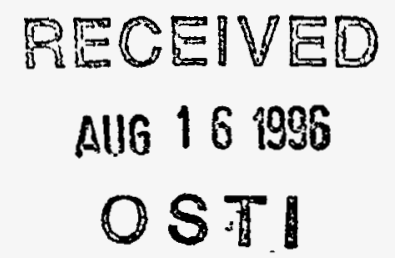

\section{Abstract}

One of the more bothersome aspects of developing a parallel program is that of monitoring the behavior of the program for debugging and performance tuning. Often there is no intrinsic support for examining program state and dynamics in a parallel programming system, let alone a useful interface for analyzing or visualizing that information. This paper discusses an enhanced tracing facility and tracing tool for PVM (Parallel Virtual Machine), a message passing library for parallel processing in a heterogeneous environment. PVM supports mixed collections of workstation clusters, shared-memory multiprocessors, and MPPs. The upcoming release of PVM, Version 3.4, contains a new and improved tracing facility which provides more flexible and efficient access to run-time program information. This new tracing system supports a buffering mechanism to reduce the perturbation of user applications caused by tracing, and a more flexible trace event definition scheme which is based on a self-defining data format. The new scheme expedites the collection of program execution histories, and allows for integration of user-defined custom trace events. The tracing instrumentation is built into the PVM library, to avoid re-compilation when tracing is desired, and supports on-the-fly adjustments to each task's trace event mask, for control over the level of tracing detail. Along with this new tracing facility, the graphical console and monitor XPVM has been updated to provide better access to the new tracing functionality. Several new views have been implemented to utilize the additional tracing information now possible, including user-defined events. The XPYM system has also been optimized to provide better. real-time monitoring capabilities.

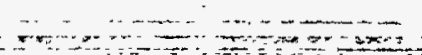

-Rescarchrsupported-1y the Applied MathemraticateSciences Research Program of the Office of Energy Research, -CH.S.D. De. partment of Energy, under contract DE-AC05-96OR22464 with Lockheed Martin Energy Research Corp.

\section{Introduction}

A wide variety of scientists and applications programmers are moving into the arena of parallel processing to solve ever larger problems with ever shorter execution time. Perhaps the greatest challenges to developing good parallel programs are successful debugging and performance tuning. The central problem is one of observability - it is difficult to determine the precise behavior of a set of concurrently executing tasks. Heisenberg uncertainty tells us that we simply cannot examine the behavior of such a system without perturbing it. Therefore, the goal of effective parallel program analysis is to provide accurate information by collecting traces with the least possible amount of intrusion on the parallel program.

This paper focuses on the tracing facility to be provided in the upcoming release of PVM (Parallel Virtual Machine) [1], version 3.4. PVM is a message passing library which supports portable, interoperable parallel processing in a heterogeneous environment. A single "virtual machine" can consist of clusters of workstations, shared-memory multiprocessors, and Massively Parallel Processors (MPPs). The tracing facility described here supports the retrieval of program state information during run-time in the form of events, which can be viewed either in "real-time" or used in "post-mortem" analyses. These "trace events" can originate from any of a heterogeneous collection of architectures, independent of the data format which is automatically converted using PVM's intrinsic data conversion techniques.

A related tool, XPVM $[2,3]$, is a graphical con-sole and trace monitor that provides a graphical interface for visualizing and processing PVM execution traces - XPYM-provides access to the PVM-censole-_commands for manipulating the virtual machine and spawning parallel-tasks, and also provides several animated yiews which monitor the status of hosts and the execution of tasks, including task to task com- 


\section{DISCLAIMER}

This report was prepared as an account of work sponsored by an agency of the United States Government. Neither the United States Government nor any agency thereof, nor any of their employees, makes any warranty, express or implied, or assumes any legal liability or responsibility for the accuracy, completeness, or usefulness of any information, apparatus, product, or process disclosed, or represents that its use would not infringe privately owned rights. Reference herein to any specific commercial product, process, or service by trade name, trademark, manufacturer, or otherwise does not necessarily constitute or imply its endorsement, recommendation, or favoring by the United States Government or any agency thereof. The views and opinions of authors expressed herein do not necessarily state or reflect those of the United States Government or any agency thereof. 


\section{DISCLAIMER}

Portions of this document may be illegible in electronic image products. Images are produced from the best available original document. 
munication. These views provide information about the interactions among tasks in a parallel program, to assist in debugging and performance tuning. XPVM is the next generation of a previous system known as Xab $[4,5]$. XPVM encompasses the functionality of the Xab system and extends it, and in addition uses the intrinsic PVM tracing facility rather than the custom Xab tracing instrumentation ${ }^{1}$.

The upcoming release of PVM, version 3.4, contains an enhanced tracing facility which provides better flexibility and efficiency in gathering traces. The new facility supports buffered tracing, such that a large number of trace events can be combined into a single message for transferral to a trace replay tool, e.g. XPVM. Buffering trace events reduces the intrusion to the parallel application, thereby improving the accuracy of the resulting traces. A new trace event definition scheme has also been implemented to increase flexibility and expedite the processing of traces. The new scheme provides the capability for user-defined "custom" trace events.

To better support the processing and analysis of traces generated using the new 3.4 facility, a new release of XPVM, version 1.1, is now available. XPVM 1.1 has been updated to include several new view features, and has been significantly optimized to improve its real-time monitoring and trace playback capabilities.

The remainder of this paper discusses the new PVM 3.4 tracing facility in more detail. Section 2 overviews the old PVM 3.3 tracing support for comparison purposes. Sections 3 and 4 describe the design of the new tracing facility and the new features in XPVM 1.1, respectively. Section 5 presents some experimental results comparing the new systems to the original PVM 3.3 and XPVM 1.0 releases. Section 6 proposes areas for future work.

\section{Background}

The first tracing facility for PVM was implemented in version 3.3. This tracing facility supported built-in tracing instrumentation. By integrating the instrumentation for tracing directly into the PVM library, the user application did not have to be re-compiled to affect changes in the tracing output. The destination for trace event collection, and the choice of which PVM routines to be traced, could all be selected at run-time. The new PVM 3.4 tracing facility sustains this integrated functionality - Each-trace event-captured-in-PXM-corresponds to- either the entry or exit point of a PVM library routine, and contains information about any calling parameters or returned results. The trace events are transferred to a tracing tool such as XPVM using the regular PVM message channels. XPVM receives and processes these trace event messages to produce a "trace file" which can then be read in to drive graphical or textual views. The trace files for both the original and enhanced tracing facilities are written in SDDF (SelfDefining Data Format) [6] as designed for the "Pablo" system [7]. SDDF was chosen over the PICL format [8], as used by the widely known "ParaGraph" system $[9]$, , because PICL was not sufficiently flexible to represent all the trace information generated by PVM. SDDF is a de facto standard in the program visualization community, and presents a general trace format, allowing arbitrary data structuring and including semantic information.

The original tracing facility provided with PVM 3.3 did not support any buffering mechanism, resulting in high tracing overhead. For example, each message sent between PVM tasks generated four trace event messages to XPVM, two for the send and two for the receive. This deluge of trace messages severely perturbed communication-intensive applications, slowing down their execution and making any accurate performance evaluation impossible.

Trace buffering as such is not a new concept, and many trace instrumentation systems have supported buffering as a means for reducing intrusion. However, there are several logistical concerns in designing a buffered tracing system. For systems such as PVM which often generate a continual progression of "patch levels" to fix or extend the system's functionality, proper buffer unpacking can be challenging. Each new PVM patch is an opportunity for potential changes in trace event contents, and each such change requires that the corresponding event unpacking code in XPVM be correctly adjusted - a task that could easily be overlooked. Another issue arises with userdefined events, where the user could incorrectly specify an event's contents, leading to incorrect unpacking. If some event in a trace buffer is not correctly unpacked, there may be extraneous data left in the buffer before the next event, making it impossible to confidently locate the start of that event. Alternately, too much data could be unpacked for the first event, leaving only a portion of the next event-dangling in the buffer. In eithêr case, it will be impossible to continue correctly unpacking-the given-trace-buffer-and-the:remainder of -trat=büferes=contents" will=be "lost" or-corrupted.

One of the central problems with the original PVM 
3.3 tracing facility was that the contents of trace events were hard-wired into the PVM library by hand. This made it necessary to manually code the unpacking of several trace events, opening many opportunities for error. In addition, incorporating changes in trace event contents resulted in significant bookkeeping problems for XPVM when interoperating with different patch levels. The resulting lack of stability in trace processing made the prospect of trace buffering problematic, and inspired the development of the more flexible scheme described in Section 3. This scheme supports automatic generation of trace event descriptions for both system and user-defined events, to enforce correct unpacking.

\section{PVM 3.4 Tracing Facility}

The goal of the new tracing facility for PVM 3.4 was to fix the shortcomings of the 3.3 tracing facility, and as much as possible plan for the future of PVM tracing needs. The primary features of the new facility are trace buffering, user-defined events, and flexible event contents. All of these are made possible through use of a new trace event structure. This structure includes self-defining formatting information that allows automated unpacking, as discussed in the following subsections.

\subsection{Trace Event Structure}

The new trace event structure has been designed with flexibility in mind. The approach is to explicitly define each trace event record at run time, before its appearance in an event trace. This closely models the SDDF trace file format by including "trace descriptors" in the event stream. These descriptors precisely define the contents of each "trace record," and specify the data types and unpacking order of the trace information. In addition, some semantic identification is included to assist tools like XPVM in finding and processing the raw trace data for driving animated views.

Using trace descriptors alleviates the need for including formatting separators in trace records to enforce correct unpacking. Rather than repeatedly sending this formatting information inside each event record instance, a single descriptor can be sent once as needed, and then used as a template to correctly unpack each trace record for the given event. The trace records can-then contain only the-necessary-rawdata Clearly it in inportant that the trace descriptors themselves are correctly generated. In the best cáse; these-cescriptors slould be created aútömatically to avoid any omissions or errors. This is the case in PVM 3.4.

\begin{tabular}{|c|}
\hline <Event Descriptor Marker \\
\hline "Event Name" \\
\hline Event ID\# \\
\hline Data ID / Data Type \\
\hline Data ID / Data Type \\
\hline$\vdots$ \\
\hline <Event Descriptor End Marker> \\
\hline
\end{tabular}

Figure 1: Trace Descriptor Format

The format of trace descriptors is shown in Figure 1. As with all the various trace message units in PVM 3.4, integer markers are packed at the beginning and end of each functional unit to identify the unit and separate it from other units in case events are buffered. Note that the end markers are not strictly necessary, but greatly simplify the task of unpacking a trace buffer and detecting errors.

The trace descriptor unit contains a character string "Name" describing the event, as well as an integer "Event ID" number. The Event ID is used to streamline the processing of subsequent event record instances by avoiding string lookup. Note that Event IDs are unique only within the scope of any single task, and different tasks might associate different IDs with any particular event. A trace tool such as XPVM must keep track of which Event IDs a particular task is using.

The remainder of the descriptor is a list of "Data ID / Data Type" pairs that describe the individual pieces of trace data. The "Data ID" is really a semantic identifier that indicates the origin of the particular trace data and so assists trace tools like XPVM in being able to properly interpret and present the data. The ID is a 3 -byte (character) mnemonic name that is associated with a full character string description of the data item, such as "[SRC / Message Source Task ID]." These IDs must remain fixed across all patch levels and major revisions of the tracing facility, to allow consistent identification of desired trace data. New semantic IDs can be added, but no changes or new interpretations of existing IDs can be applied. It is the semantic identifier that allows trace record contents to change and be reordered without affecting XPVM's capability to process trace data. Trace event records can be searched for any desired piece of information using-the-semantic PDs:-

As an aside, to allow some extensibility for future PVM releases, an additional trace event unitexists to define the above semantic identifiers. This unit can bêm used to define new semantic identifiers as well as to as- 


\begin{tabular}{|c|}
\hline <Event Data ID Marker \\
\hline Data ID \# \\
\hline "Data Description" \\
\hline Data ID \# \\
\hline "Data Description" \\
\hline$\vdots$ \\
\hline <Event Data ID End Marker> \\
\hline
\end{tabular}

Figure 2: Semantic Identifier Unit Format

sociate a modified character string description with an existing semantic ID. (Note that changing the description text might be applied for clarification of meaning or for special trace file notations, but should not imply an actual semantic change lest backwards compatibility be lost.) The format of the semantic trace unit is shown in Figure 2. The unit consists simply of a list of mnemonic ID / description pairs.

The data type information included in trace descriptors is encoded in a single byte, so as to fit with a semantic ID into a single word. This is done both for convenience and efficiency. The possible data types supported are shown in Figure 3. Most of these data types are self explanatory, however a few are specialpurpose in nature. The "Null" type indicates the absence of data and corresponds to an empty trace data marker. This may be used for special trace tool directives or other custom needs. The "Structure Start" and "Structure End" types are to be used in future extensions which will allow structured data to be defined and passed in trace events. Like the Null data type, these types will not correspond to real data, but will act only as markers around a structure definition consisting of a sub-list of data types. The "Deferred" type is intended for any trace data which may be of a variable data type depending on the particular run-time calling parameters of the traced routine. For deferred data an additional data type identifier is packed with the actual data in each trace record event instance.

Note that the above data types require only 4 bits to specify, leaving an additional 4 bits for extensions. One of these bits has already been reserved for determining whether the trace data is scalar or an array, as shown in Figure 4. Arrays are considered onedimensional for simplicity, hence the number of dimensions need not be specified. For the purposes of passing-trace-information there-is-as yet no-ned-for-multi dimensional arrays, as any multi-dimensional array can be unrolled into a simple one-dimensional vector:

$\therefore$ Should the need arise for true multi-dimensionalarray data, either in user-defined events or for some future

\begin{tabular}{|l|r|}
\hline Null & 0 \\
\hline Byte & 1 \\
\hline Complex & 2 \\
\hline Double Complex & 3 \\
\hline Double & 4 \\
\hline Float & 5 \\
\hline Integer & 6 \\
\hline Unsigned Integer & 7 \\
\hline Long Integer & 8 \\
\hline Unsigned Long & 9 \\
\hline Short Integer & 10 \\
\hline Unsigned Short & 11 \\
\hline String & 12 \\
\hline Structure Start & 13 \\
\hline Structure End & 14 \\
\hline Deferred & 15 \\
\hline
\end{tabular}

Figure 3: Descriptor Data Types

\begin{tabular}{|l|l|}
\hline Data Scalar & $0 \times 00$ \\
\hline Data Array & 0x80 \\
\hline
\end{tabular}

Figure 4: Descriptor Data Type Dimension

tracing feature, the dimensions and indices into such an array could easily be packed with the array to allow proper decoding of the one-dimensional data vector.

Once a trace descriptor for a particular event has been sent, the corresponding trace records can then be sent and properly unpacked. This unpacked trace data is typically placed into a trace file or interpreted to drive analyses or animations. It should be noted that all trace messages in PVM are packed and sent like any other PVM messages, using the standard PVM message channels. XPVM is itself a PVM task, with a regular PVM task ID, and trace messages are directed to XPVM using this task ID and a special message code that XPVM chooses.

The format of trace record events is very simple, consisting only of an event ID number and the trace data itself. This format is shown in Figure 5. The "<Event Data Marker>" and "<Event Data End Marker>" markers are present to verify the boundaries among events in a buffer. As stated above, not all markers are strictly necessary, but they can be employed to simplify the unpacking of a trace event buffer. They are also a handy safeguard and do not incur significañit overead.

\section{2--Trace-Descriptor-Issues}

In PVM 3.4, trace descriptors are constructed when the $P V M$ library is compiled, not at run time. The descriptors are automatically generated by pre-parsing 


\begin{tabular}{|c|}
\hline <Event Record Marker> \\
\hline Event W \# \\
\hline \{ Event Data... \} \\
\hline <Event Record End Marker> \\
\hline
\end{tabular}

Figure 5: Trace Record Format

the code that actually packs the trace records. In this way, there is significantly less chance for any discrepancy between corresponding descriptor / record pairs. A direct result of this is the capability for reliable trace event buffering.

There is, of course, some overhead associated with sending the additional trace descriptor events. But, with trace buffering it is possible to "piggy-back" each descriptor with the first instance of each corresponding trace record, thereby incurring little additional message overhead. Also, because the descriptors are already constructed at compile time, a single conditional can be used to trigger the descriptor - i.e. every time an event occurs, a simple flag is checked to determine whether the descriptor has been sent for that event yet.

Several alternate methods exist for communicating the trace descriptors, but they each have significant drawbacks. The descriptors could be constructed onthe-fly at run-time instead of at compile time, to avoid the pre-parsing step. But if the descriptors were generated at run-time, the only reliable means for creating them would require monitoring each piece of data as it was packed into the event record. This would require some sort of conditional check for each piece of event data, every time it was packed! In addition, this method would result in significant complexity and bookkeeping from trying to generate the descriptor and record simultaneously.

Likewise, another alternative uses a special combination descriptor-record, with both formatting and data "merged" together into one trace unit. This approach would also incur the repetitive overhead above, long after the descriptor information was sent, as the same conditionals would be executed for each piece of event data, again and again.

\subsection{Trace Facility Administration}

There are other benefits to this tracing facility approach; in terms of mainteñance, interòperability and extensibility. Because each task sends its.own descriptors, it is possible for tasks running a yariety of vendor and public PVM distributions, at-different patchlev= els, to:-intcroperate-in-the-same-program-trace-Tools such as XPVM need not endure the hassle of extensive bookkeeping to determine the various trace record contents for all different versions, but instead can just maintain a run-time list of the current descriptors being used by tasks for the given trace. New trace events can be added and trace record contents rearranged without modifying XPVM's trace file generation code. The new events may not yet be interpreted, but the trace data can be saved so a future version of XPVM will be able to use it. Also, any specifications of view functionality can be implemented in a generic manner using the semantic identifiers and event names. This raises the possibility that some new events could be automatically interpreted and used in views based solely on their semantic content.

\subsection{Trace Buffering}

With the above new event structure, trace event buffering becomes a straightforward and safe feature to provide. Arbitrarily large collections of trace events can be correctly unpacked using the well-defined trace descriptor format information. Trace buffering in PVM 3.4 consists of packing multiple event units into the same PVM message buffer. Rather than sending many small event messages, one large message can be sent to reduce intrusion on the user application. This improves the accuracy of the tracing and the convenience to the user (in terms of reduced overhead).

The user interface to trace buffering in PVM 3.4 uses the existing PVM options routines, pvm_setopt () and pvm_getopt(). A user application can manually set its level of trace buffering using a call like:

$$
\text { pum_setopt ( PrmTraceBuffer, size); }
$$

where the size argument can be an arbitrary positive integer, or zero to buffer the entire execution's trace events. Note that in cases where the entire execution is to be buffered, care must be taken to insure that sufficient memory is available to hold the entire buffer of trace events. It is perhaps wiser for most full-scale applications to choose some large buffer size rather than buffering the entire execution. Also note that, like task output or any buffered data, a PVM task must exit normally using pvm_exit () for any remaining trace buffer to be obtained by XPVM. Otherwise, there is no reliable way to invoke the sending of the last-trace buffer and it will be lost.

Typically, the user_will_not need_to manually set - the level of trace buffering within the application, but can rely on XPVM to handle this function at run time. When tasks are spawned from XPVM, they are set to trace certain - Toutines and-the-trace-buffering is set. The settings for the-trace mask and the trace-buffor 
size are both set initially from the XPVM Spawn dialog, but XPVM version 1.1 also supports "on-the-fly" adjustments to various tracing parameters, including the trace buffer size.

\subsection{User-Defined Trace Events}

Another useful feature made possible with trace descriptors is the capability for the user to define custom trace events. Custom events make the tracing facility extensible for any special user needs. In addition, they provide a means for the advanced PVM user to determine the behavior of an application outside the scope of PVM system calls. While the remainder of the tracing facility is solely designed for providing information about the invocations of the PVM library routines, user-defined trace events allow the user to collect information about the actual computation that is using PVM. With user-defined trace events, a programmer can send back intermediate results or mark the passing of simple atomic occurrences, such as phases of a computation.

For simplicity, the interface for custom trace events precisely resembles the regular PVM message interface. A special message encoding "PvmDataTrace" has been added, so that the user can use the existing pvm_pk*() routines to manually pack up an event as easily as any other PVM message. An example custom event is shown in Figure 6. The PvmDataTrace encoding automatically inserts sufficient data typing information in the message to allow correct unpacking of the event by a tool such as XPVM. If trace buffering is in effect, the message will be appended directly to any existing trace buffer. After the event is packed, pvm_send () is called with special predefined constants for the trace collection destination task ID and tracing message code. These constants dereference to the correct internal tracing destination, e.g. XPVM. If the trace buffer is full or buffering is not activated, then the pvm_send() call will flush the custom event, along with any other buffered events. Otherwise, the custom event will wait in the buffer for a future buffer flush.

Like other trace events, user-defined events can be controlled by setting the PVM trace mask. A single trace mask entry can be used to activate or deactivate all the user-defined trace events in an application. This control can be exerted at run time without recompilation, either at spawn time or on-the-fly using XPVM.If tracing is not activated or the trace mask for-the -user-defined-events-is-not-set;-then-the-PAAT packing and sending routines will become NOP, and will have.no.effect....

If XPVIV is'being used"to collect trace events, then pvm_initsend( PvmDataTrace); pvmpkstr( "PVH is Cool, Start Phase III"); pvm_pkint ( \&my_int_array, 10, 3);

pvm_pkdouble ( \&my_double, 1, 1 ); pvm_send( PVH_TRACE_TID, PVM_TRACE_CODE );

Figure 6: Custom Trace Event Generation

with the other regular events. A special "tick" mark will appear on the Space-Time View, and a textual representation of the data sent will appear in the Call Trace View. It is up to the user to decide how to interpret and use the information returned from userdefined events.

\section{XPVM 1.1}

To fully support the new features and information available in the PVM 3.4 tracing facility, XPVM has been upgraded to a new version 1.1. This version includes a new Message Queue View and enhancements to several existing views. This version has also been optimized and re-organized to improve the "friendliness" of its user interface. The resulting interface is shown in Figure 7.

XPVM 1.1 is fully backward compatible with the previous PVM 3.3 tracing facility event formats, and can simultaneously handle either format. PVM 3.3 and 3.4, however, support sufficiently different message passing protocols that XPVM must be compiled for a specific PVM version to receive its trace event messages. Note however that any message-compatible PVM (e.g. vendor versions) can interoperate, independent of trace format.

XPVM 1.1 is now fully compatible with both sets of the TCL and TK tool language systems. Some optimizations have been applied to XPVM 1.1 as well. Because the interpreted TCL and TK languages adversely affected the performance of XPVM 1.0, much of the functionality has been migrated to faster $\mathrm{C}$ code. This has resulted in an order of magnitude improvement in many code segments.

The following subsections describe the new view features in XPVM 1.1 in more detail.

\subsection{Network View Enhancements}

The Network View is a depiction of the high-level activity on the hosts in the virtual machine, with colored icon images that are animated according to the status of each host__One of the drawbacks of the origi-

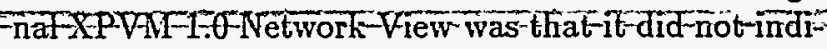
cate the load of the network or its-performance. The new_Network View:- contains animated depictions.of _....... the instantaneouis network bandwidth and volume on -.............. -individual-network-links. - . 


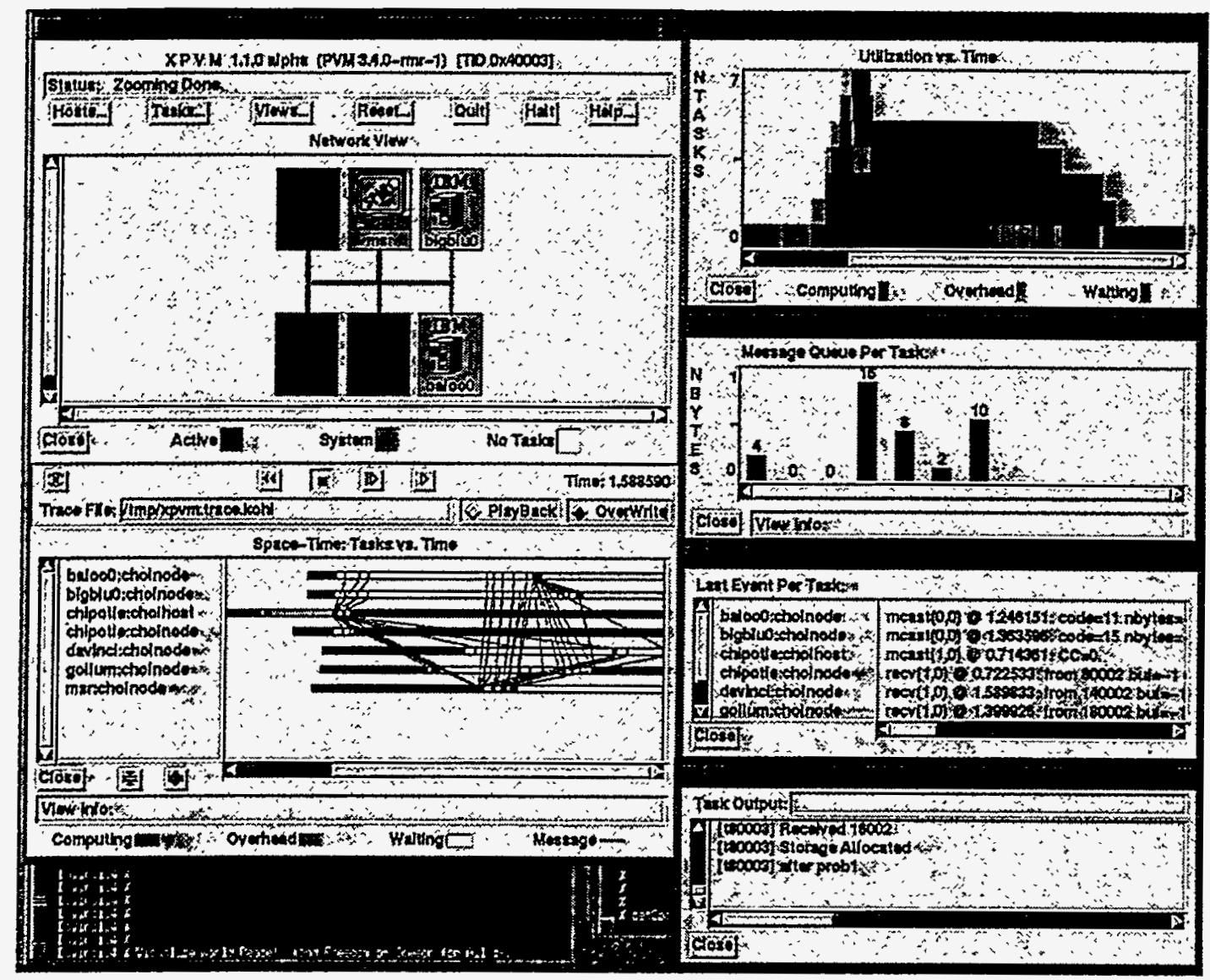

Figure 7: XPVM 1.1 Interface

The primary network topology supported in XPVM 1.1 is a simple bus, as typically seen with Ethernet networks. At present all topologies are mapped to the this bus layout. The links from each host to the bus, and the individual bus links, are all animated. The width of a particular link corresponds to the bandwidth most recently realized on that link, and the message volume is shown by the color. An example of the new Network View is shown in Figure 8.

The bandwidth is calculated upon message receipt, because it is not known in advance how long a particular message will take to be transmitted. Specifically, the bandwidth is the average communication rate in bytes per second, equal to the ratio of total message size to total travel time. The bandwidth can be used to determine the load and performance of the network, thereby providing insight into the impact the network is having on the application's performance.

Thë-message yolume represents a wide spectrum of ranges from bytes to kilobytes to megabytes. The volüme is computed on-the-fly by adding and-subtracting the size of messages as they enter and leave the network. This information provides an indication of the existence and magnitude of any communication bottlenecks in the application.

\subsection{Utilization View Optimizations}

The Utilization View shows the cumulative status of all tasks executing at each time instant. The view is depicted by a long line of histogram stacks, three rectangles per stack, spread along a horizontal time axis. At each time, the three rectangles represent the number of tasks that are busy computing, in system overhead and idle blocked on communication, respectively.

In the previous version of XPVM 1.0, the Utilization View was the most computation-intensive view animation. The problem came from the fact that events were often collected over a network. Due to varying travel times, event messages from some hosts could arrive "behind schedule" compared to other hosts, resulting in an unfortunate "re-calculation" of the task utilization states. Events could arrive arbitrarily far back "in the past," requiring adjustment of the utilization task counts from that time up though the current time. This repeated reccalculation of the utilization history became an immense performance

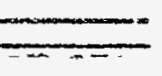


problem, especially with large traces.

In XPVM 1.1, the solution is a slight modification of the Utilization View logic. The original Utilization View assumes a continuation of the last recorded state for a task until the next event is received. Thus, the right leading edge of the view progresses as if the state of all tasks is known. In the optimized view, a task's status is not added to the utilization task counts until that task's state is known up to a particular time. This results in a "progressive" leading edge as tasks gradually fill in their events from left to right, as shown in Figure 9. The overall result is a more correct depiction of the true task utilization (without any recursive "recalculations"), that is also significantly more efficient by several orders of magnitude.

\subsection{Message Queue View}

The Message Queue View is a new view introduced in XPVM 1.1. This view shows the instantaneous number and size of messages that are buffered, waiting to be received, for each task. The view consists of a collection of "message stacks," one for each task, that represent the pending messages. Each stack is made up of rectangles that represent the individual pending messages. This information is especially useful in identifying communication "hot spots," much like the message volume animation in the enhanced Network View, but at a higher resolution. In this view the message bottlenecks among individual tasks can be observed. The view is also "clickable" to reveal detailed textual information for individual message rectangles. This information includes the source task of the message, the message code, and the number of bytes (if known, see below). A sample of this view is shown in Figure 10.

It should be noted that development of the Message Queue View required the flexibility of the new tracing facility in PVM 3.4. To depict the number of bytes in pending messages, their size must be known at send time. In the original PVM 3.3 tracing facility, this information had been omitted, and so precluded the use of the message size. The PVM 3.4 tracing facility was required to add the size of the outgoing message to the send event data. (For compatibility with PVM 3.3 tracing, a default message size can be set using the "-M size" command line option to XPVM. To distinguish the default message size from actual message sizes, different colors are used for the message-rectangles.) collected. A parallel Cholesky matrix factorization algorithm was applied for the timings, using a 100x100 matrix. The results of these timings are presented in the following subsections.

\subsection{Enhanced PVM Performance}

The execution time of the PVM Cholesky application was measured for both the old PVM 3.3 and new PVM 3.4 tracing facilities, with all possible trace events activated. For the PVM 3.4 timing runs, three different trace buffer sizes were used, for $1,8,20,100$ and 1000 buffered trace events. The results are shown in Figure 11. For PVM 3.3 tracing, the Cholesky example took over 33 seconds with tracing versus only 8 seconds without tracing, for a $400 \%$ tracing overhead. For PVM 3.4 tracing with no trace buffering (buffer size set to 1), comparable results were found. Only a minor additional degradation was seen in the PVM 3.4 tracing due to the additional trace descriptor information.

When trace buffering is applied in PVM 3.4 tracing, a significant improvement is seen. For a buffer size of 8 , the execution time drops to about 23 seconds, for less than $300 \%$ overhead (a $25 \%$ improvement). Ultimately, for a buffer size of 1000 the tracing overhead becomes almost negligible, with an execution time just a bit over 8 seconds, for a total overhead of only $2-3 \%$.

\subsection{Optimized XPVM Performance}

To examine the improvement in efficiency in XPVM 1.1, a sample trace from the above PVM timings was run through both XPVM 1.0 and 1.1. The timing differences were staggering, as shown in Figure 12. For a large trace file of 0.5 Megabytes, representing 34 seconds of execution, XPVM 1.1 animated the trace in exactly 2 minutes with the Network and SpaceTime Views activated. Playing the same trace through XPVM 1.0 was infeasible, taking 30 minutes for only the first 6 seconds of trace time. Projecting from this initial timings, the total time to animate the trace in XPVM 1.0 is well over 2.5 hours. This is a difference of 75 times, or almost 2 orders of magnitude.

For the same trace run with all views activated (including the extra Message Queue View not available in XPVM 1.0), the XPVM 1.1 animation took only 191 seconds. The XPVM 1.0 animation only reached 4 seconds of trace time after 30 minutes, for a difference of over 80 times or two orders of magnitude improvement.-

\section{5 -Results}

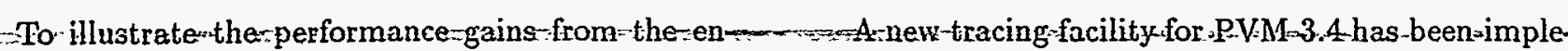
hanced-PVM tracing-facility,-as-well-as-the-optimizations to XPVM, a representative set of timings was mented=for more-flexible-and efficient access-to-runtime parallel program information. The new tracing 


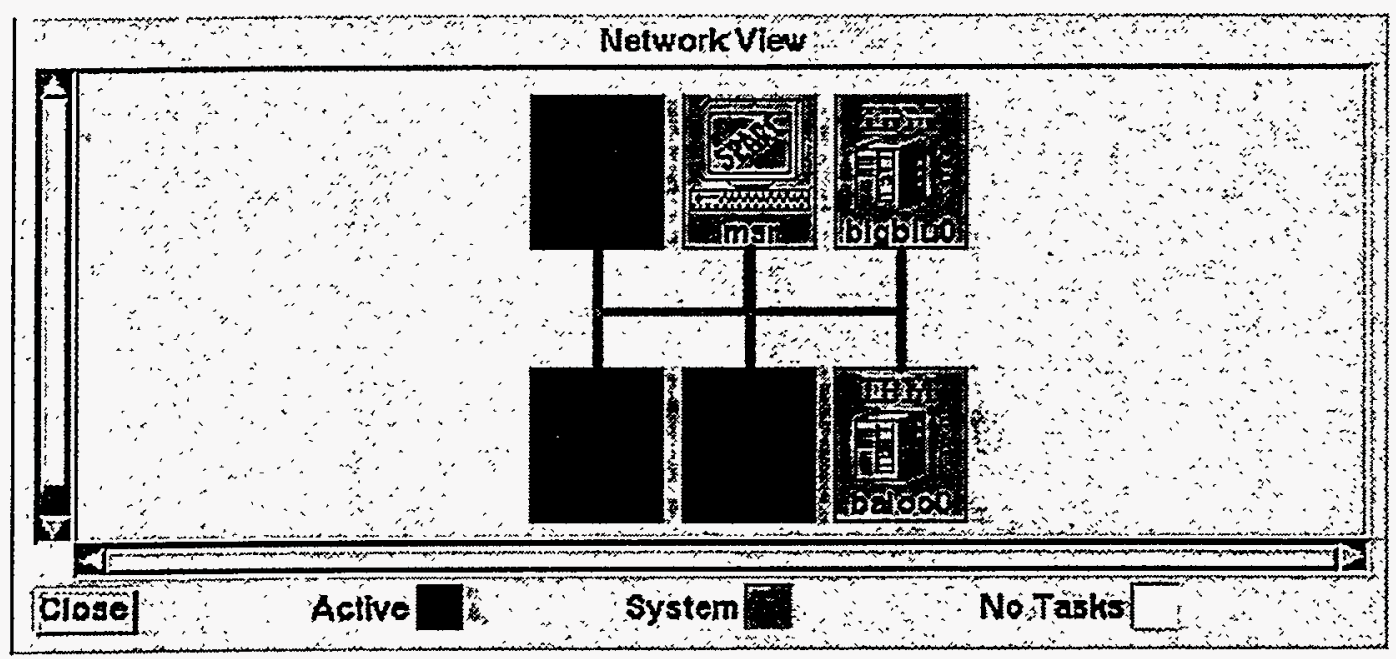

Figure 8: Enhanced Network View

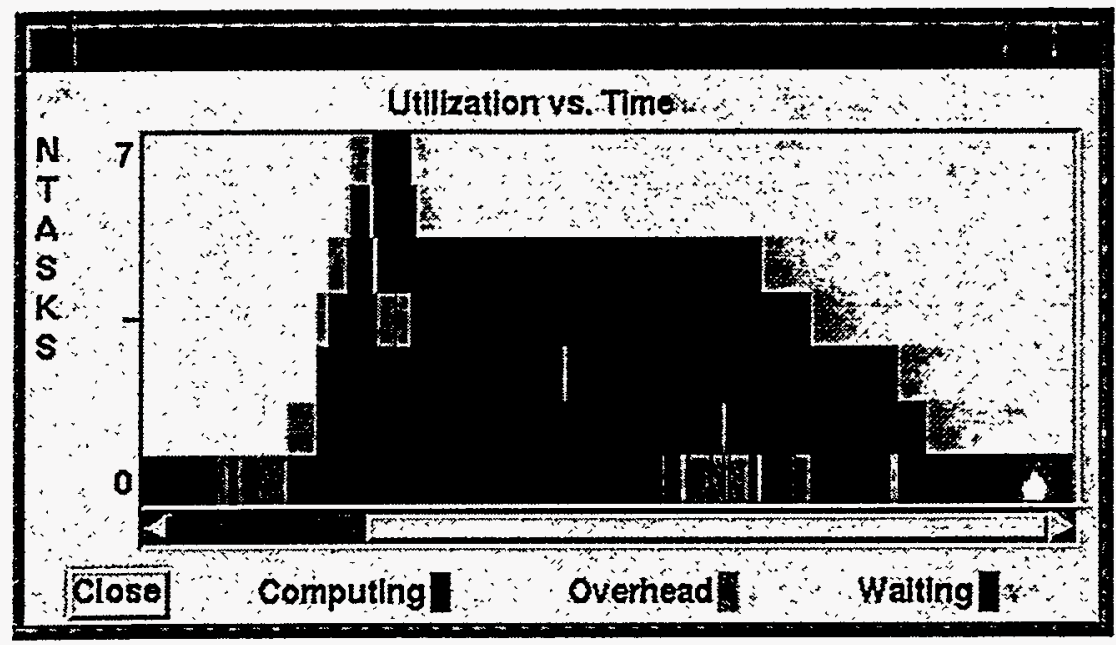

Figure 9: Optimized Utilization View

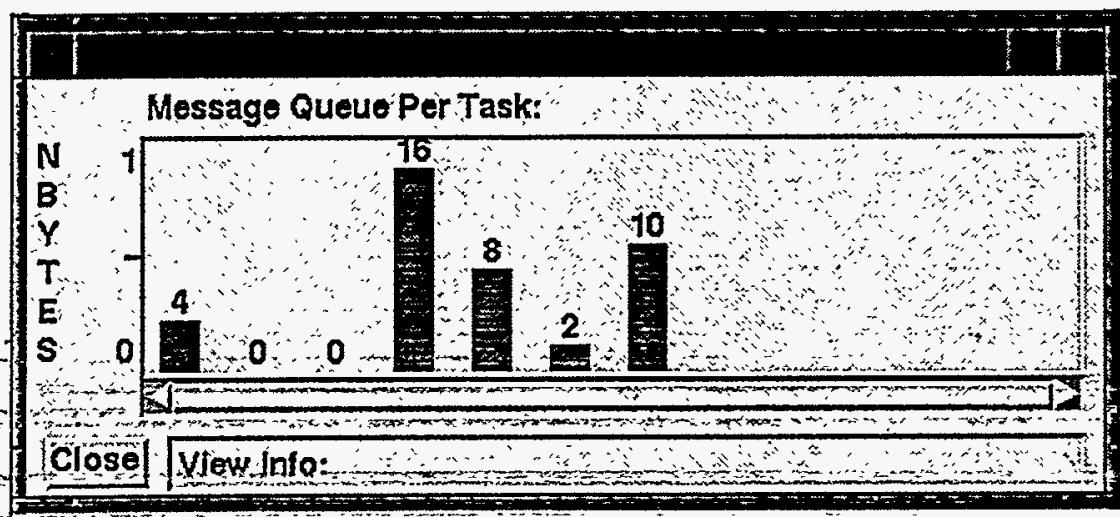

Figure 10: New Messagè Queue View 


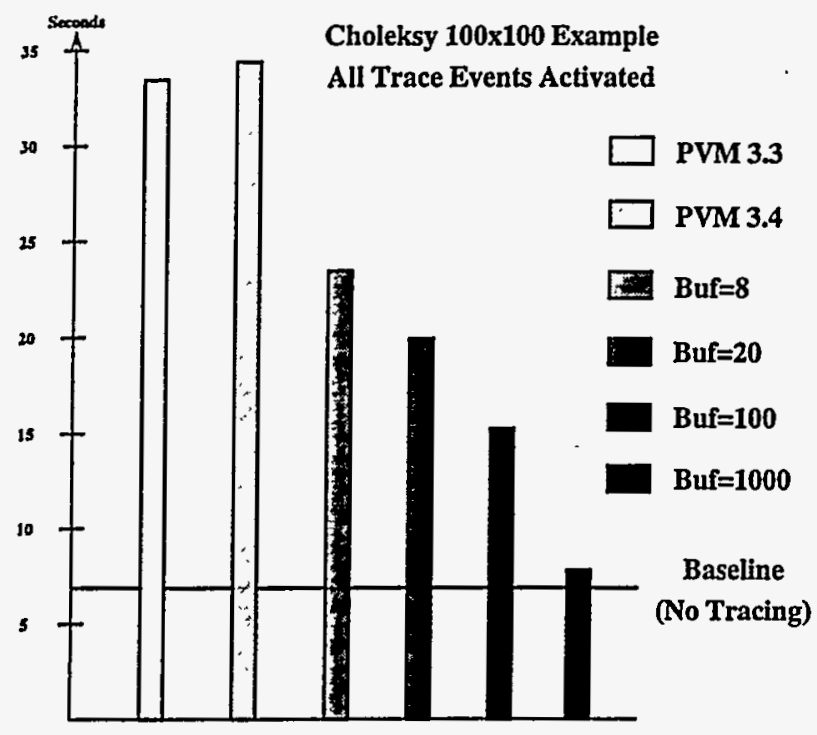

Figure 11: PVM Tracing Facility Comparison

system supports buffered tracing to reduce the perturbation of tracing user applications, thereby improving the accuracy of trace information for debugging and performance tuning. The new system also supports custom user-defined events. In conjunction with the new PVM 3.4 tracing, an enhanced version of XPVM 1.1 has been developed to utilizes the new tracing information and provide new and extended view animation. Both PVM 3.4 tracing and XPVM 1.1 have been shown to perform substantially more efficiently in collecting and animating parallel program traces.

There are several directions for future work. There is a need for higher level (less detailed) tracing information for more effective performance tuning and other general analyses. The immense amount of information from parallel program execution makes it critical to find ways to reduce the size of execution traces.

It is also essential to continue searching for better ways to visualize the mountains of trace data already collected, so that users can more readily understand the behavior of interest. Tools need to start assisting users by pointing out anomalous behavior which may be causing problems, yet may not be obvious or expected by the user. The wealth of existing statistical analysis techniques should be applied to this end.

\section{References}

[1] G. A. Geist, A. Beguelin, J. Dongarra, W. Jiang, R. Manchek V. Sunderam; PVM-Parallel-Virtual Machine, $A$ User's Gude and Tutorial for Networked -Parallel Gomputing-The-MIT-Press;-1994.

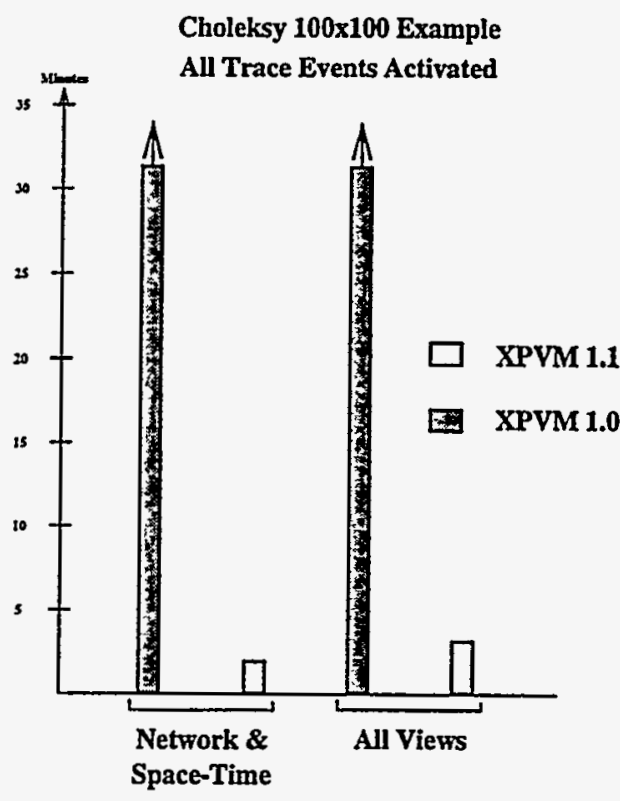

Figure 12: XPVM Efficiency Comparison

Computer Science and Mathematics Division, Oak Ridge National Laboratory, Oak Ridge, TN, April 1995.

[3] A. Beguelin, J. Dongarra, A. Geist, R. Manchek and V. Sunderam, "Recent Enhancements to PVM," The International Journal of Supercomputer Applications and High Performance Computing, 9(2), pp 108-127, Summer 1995.

[4] A. Beguelin, "Xab: A Tool for Monitoring PVM Programs," Workshop on Heterogeneous Processing, April 1993, Newport Beach, California. pages 92-97, IEEE Computer Society Press.

[5] A. Beguelin, J. Dongarra, A. Geist, and V. Sunderam, "Visualization and Debugging in a Heterogeneous Environment," IEEE Computer, 26(6), pages 88-95. June 1993.

[6] R. A. Aydt, "The Pablo Self-Defining Data Format," University of Illinois at Urbana-Champaign, Department of Computer Science, February 1993.

[7] D. Reed, R. Olson, R. Aydt, T. Madhyastha, T. Birkett, D. Jensen, B. Nazief, B. Totty, "Scalable Performance Environments for Parallel Systems," Proceedings of the Sixth Distributed Memory Computing Conference, IEEE Computer Society Press, April 1991.

[8] P. H. Worley, "A New PICL Trace File Format," Technical Report ORNL/TM-12125, Oak Ridge Nàtional Laboratory, Oak_Ridge, TN October 1992.

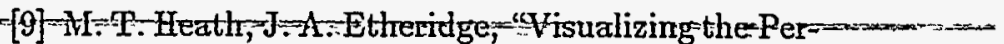
formance of-Parallel-Programs, "IEEE-Software,_______ Volume 8, Number 5, September 1991, pp. 29-40.

Guide," Technical Report ORNL/TM-12981, 